\title{
The Mating-type Systems of the Myxomycetes Physarum rigidum and $P$. flavicomum
}

\author{
By MARY R. HENNEY AND H. R. HENNEY, JUN. \\ Department of Biology, University of Houston, Houston, Texas, 77004, U.S.A.
}

(Accepted for publication I8 April I968)

\begin{abstract}
SUMMARY
Three geographical collections (isolates) of myxomycetes originally identified as Physarum flavicomum were found to be heterothallic and the clones established from each isolate were divided into two mating types. Previous studies of six isolates of $P$. flavicomum showed that they could be divided into three groups. Mating occurred within groups but not between groups, and within each group there were two or more mating types. When the new isolates were crossed to the original isolates, they conformed to two of these three groups. This study established the existence of multiple alleles at the incompatibility locus in group 3. Mating-type alleles of the $F_{1}$ generation of group 3 segregated in a $I$ : I ratio at meiosis. Isolates belonging to group I were also separated from the other two groups on morphological and physiological bases. The morphological description fits that of $P$. rigidum more nearly than $P$. flavicomum and group I isolates were placed in the species $P$. rigidum. Groups 2 and 3 were separated genetically and physiologically but morphologically the separation was not distinct. These groups were referred to as $P$. flavicomum varieties $\mathbf{I}$ and 2 .
\end{abstract}

\section{INTRODUCTION}

The life cycle of the myxomycetes (Alexopoulos, 1963) is initiated by the liberation of myxamoebae from the haploid spores. The myxamoebae divide mitotically and when a large population has formed two myxamoebae fuse to form a diploid zygote. Karyokinesis continues but cytokinesis fails to take place resulting in a large multinucleate plasmodium. Under certain environmental conditions fruiting bodies are formed; meiosis takes place with the formation of the haploid spores. In heterothallic myxomycetes the two myxamoebae that fuse to form the zygote must be of opposite mating types and come from different spores.

The list of heterothallic myxomycetes has grown considerably since Dee (I960), with Physarum polycephalum, first established the existence of an incompatibility mechanism in this group of organisms. These include Didymium iridis (Collins, 1961), $P$. pusillum (Collins, 1962), Fuligo septica (C. J. Alexopoulos \& M. R. Henney, unpublished data), P. globuliferum(M. R. Henney, unpublished data), Comatricha laxa (Wollman, I966), and P. flavicomum (Henney, 1967). It has further been shown that both homothallic and heterothallic isolates occur in the same species and that these isolates are indistinguishable on morphological grounds. The term 'isolate' designates a specimen collected at a specific geographical location at a specific time. Collins (I965) reported a homothallic isolate of $D$. iridis. C. J. Alexopoulos \& M. R. Henney (unpub- 
lished data) found three of five isolates of $P$. pusillum to be heterothallic and the other two to be homothallic. They found similar results while working with $F$. septica, four of seven isolates were heterothallic and three were homothallic. This could explain the contradictory results obtained by von Stosch (1935), who reported D. nigripes to be heterothallic, and Kerr \& Sussman (1958), who reported the same organism to be homothallic.

The existence of multiple alleles at the incompatibility locus has been reported in heterothallic myxomycetes. This has been established for Didymium iridis (Collins 1963), Fuligo septica (C. J. Alexopoulos \& M. R. Henney, unpublished data), Physarum polycephalum (Dee, I966), and $P$. flavicomum (Henney, 1967). Six isolates of the latter (each consisting of two mating types) could be divided into three groups based on their ability to cross with one another. Members of the first group crossed with each other in such a way as to indicate the existence of six different mating types. Members of the second group again crossed with other members of their group, indicating four mating types. The sixth isolate could be divided into two mating types that would not cross with the remaining five isolates. These six isolates could also be divided into three corresponding morphological groups based on spore size, shape of sporangium and stalk length. Further studies of three more isolates originally identified as $P$. flavicomum have been conducted and compared to the six original isolates (Henney, I967).

\section{METHODS}

Cultures. The three new geographical collections (isolates) were all found in Texas and are referred to as TEXAS III, TEXAS IV and TEXAS V. Isolates TEXAS III and TEXAS IV were collected by the authors in Bastrop State Park near Austin, Texas, November 1965. These isolates were collected at the same time and within a few yards of one another. The TEXAS $\mathrm{v}$ isolate originated in a moist chamber composed of debris collected near San Antonio, Texas, 1964, and was kindly supplied by Dr Constance Wollman. The sources of the original six isolates, TEXAS I, PHILIPPINE I, COSTA RICA, PHILIPPINE II, AFRICA and TEXAS II, are given in an earlier paper (Henney, I967).

Clonal establishment. Clones were established by distributing a suspension of spores over the surface of a plate of half-strength corn meal agar containing $8.5 \mathrm{~g}$. Difco (Detroit, Michigan, U.S.A.) corn meal agar, I2.5 g. Difco plain agar, and $1000 \mathrm{ml}$. distilled water. Under 120 magnification a single spore was isolated by cutting-out, with a fine sterile entomological needle, a small block of agar upon which it rested. The agar block bearing the spore was transferred to a $60 \times 20 \mathrm{~mm}$. sterile Petri dish containing $8 \mathrm{ml}$. half-strength corn meal agar to which was added $0.5 \mathrm{ml}$. of a dilute suspension of Aerobacter aerogenes or Escherichia coli. The single-spore cultures were incubated at $25^{\circ}$ and were examined after 2 and 4 weeks. By this time there was a large myxamoebal population in all plates where germination had occurred. Each population originated from a single spore and is referred to as a clone.

Axenic clones. Axenic and monoxenic clones were established by placing 3 or 4 drops of a myxamoebal suspension near the edge of a half-strength corn meal agar plate and tilting the plate to allow the suspension to run across the agar. The plates were incubated at $25^{\circ}$ for several days. The contaminating bacteria formed colonies all along the line of inoculation. By the end of one week the myxamoebae had multiplied and large numbers of them had crawled away from the line of inoculation and 
from the bacteria. A small agar block containing 20-30 myxamoebae was cut out and transferred to a second half-strength corn meal agar plate. Axenic and monoxenic clones were prepared by adding $0.5 \mathrm{ml}$. of a formalin-killed, or living bacterial suspension, respectively, to the block of agar containing the myxamoebae. To test the purity, five other agar blocks were cut out immediately adjacent to the original block. Each block was placed in a different bacteriological medium. The media used included glucose broth, yeast extract broth, AC broth, Bristol's solution, and thioglycollate broth (Henney, 1966). Small agar blocks were cut out from axenic cultures and placed in the above media. The media were all incubated at $25^{\circ}$ and $37^{\circ}$ for 30 days. When bacterial growth developed, as indicated by turbidity, in any of the five test media, the entire experiment was discarded and the whole procedure repeated.

Crosses. Crosses were made by inoculating 5 drops of a myxamoebal suspension, by using a sterile Pasteur pipette, from each of two different clones on to a $60 \times 20 \mathrm{~mm}$. Petri dish containing $8 \mathrm{ml}$. of half-strength corn meal agar. A living bacterial suspension, $0.25 \mathrm{ml}$., was added to each cross. All crosses were made in duplicate. In addition, duplicate cultures of the individual clones were always used as controls. Crosses were examined after I week and I month and scored + or - for plasmodial formation. Plasmodial formation was always the criterion used for compatibility.

\section{RESULTS}

\section{Intra-isolate crosses}

Thirty clones were established, as described in methods, from the TEXAS III isolate and ten of these, selected at random, were crossed in all possible combinations. The results indicated the clones were of two mating types. The remaining 20 clones were crossed to two tester clones of the two mating types selected on the basis of the first results. Eleven of the clones formed plasmodia when crossed to one of the tester clones but not with the 2nd. The remaining nine clones formed plasmodia when crossed to the 2nd tester clone but did not form plasmodia when crossed to tester clone number $\mathrm{I}$. Similar results were obtained with the TEXAS IV and TEXAS $\mathrm{v}$ isolates.

\section{Inter-isolate crosses}

Inter-isolate crosses were always made by crossing ten clones of each isolate (five of each mating type) with ten clones of every other isolate. The results of inter-isolate crosses of the original six isolates showed that three compatibility groups were formed containing three series of incompatibility alleles. Group I consisted of the TEXAS I, PHILIPPINE I and COSTA RICA isolates. Following the example of Collins (1963) the mating types of the different isolates were assigned the allele numbers $\mathrm{A}_{\mathrm{I}}$ and $\mathrm{A} 2$ for the TEXAS I isolate, A 3 and A 4 for the PHILIPPINE I isolate and A 5 and A 6 for the COSTA RICA isolate. Group 2 consisted of the PHILIPPINE II and AFRICA isolates. These two isolates crossed among themselves but would not cross with the other four isolates. The two mating types of the PHILIPPINE II isolate were assigned allele numbers B I and B 2, and the AFrICA isolate B 3 and B 4. The third group consisted of the single isolate TEXAS II. The TEXAS II isolate would not cross with any of the other isolates. The two mating types of this isolate were assigned the allele numbers $\mathrm{C} I$ and $\mathrm{C} 2$.

The inter-isolate crosses involving the three new isolates were made to see if they would fit into the existing compatibility groups or form still more groups. The results 
indicated the TEXAS $\mathrm{V}$ isolate belonged to group $\mathrm{I}$ and as both mating types of this isolate crossed with all six established alleles, the mating types were allelic to but different from those of the original isolates. These new mating types were assigned allele numbers A 7 and A 8. Previous to this study it was presumed that multiple alleles

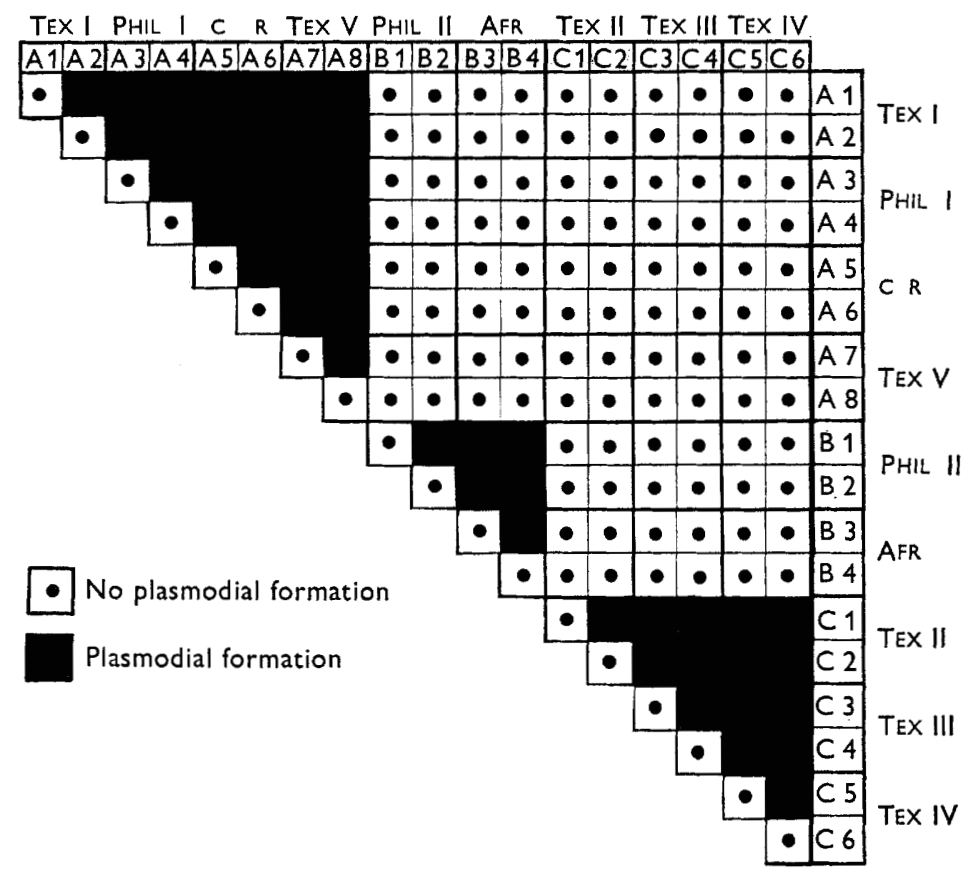

Fig. I. Physarum flavicomum. Results of the intra-isolate, inter-isolate and inter-group crosses of all nine isolates. The isolates are divided into three compatibility groups. Group I consists of the TEXAS I, PHIIPPINE I, COSTA RICA and TEXAS $V$ isolates and have been assigned mating-type alleles A I-A 8. These isolates cross among themselves but not to the other five isolates. Group 2 consists of the PHILIPPINE II and AFRICA isolates and have been assigned mating-type alleles B I-B 4 . The third compatibility group consists of the TEXAS II, TEXAS III and TEXAS IV isolates. These three isolates cross among themselves but not to the other six; they comprise mating-types alleles C I-C 6.

existed at the incompatibility locus in group 3 as well as the other two groups but this was not definitely established as only a single isolate of this group had been studied. However, when clones of the TEXAS III isolate were crossed to clones of the TEXAS II and TEXAS IV isolates it was found that both mating types crossed with both mating types of the other two isolates, proving the existence of multiple alleles at the incompatibility locus in group 3. It is especially interesting that these two isolates had different mating-type alleles as they were collected at the same time and in very close proximity. The two mating types of the TEXAS III isolate were assigned allele numbers $C_{3}$ and $C_{4}$. Those of the TEXAS IV isolate were assigned $C_{5}$ and $C 6$. The results of all the inter-isolate crosses are summarized in Fig. I. 


\section{$F_{1}$ generations}

Ten $F_{1}$ clones were established by isolating single spores from sporangia obtained by crossing two compatible clones of each isolate (intra-isolate cross). When these clones were crossed among themselves in all possible combination, in duplicate, they could again be segregated into two mating types that were inherited in a $I: I$ ratio. The

Table I. Ten clones of the $F_{1}$ generation of Physarum flavicomum TEXAS III crossed in duplicate to tester clones of the two mating types of each of the nine isolates

\begin{tabular}{|c|c|c|c|c|c|c|c|c|c|c|c|c|c|c|c|c|c|c|}
\hline \multirow{2}{*}{$\begin{array}{c}\text { Clone } \\
\text { numbers }\end{array}$} & \multicolumn{2}{|c|}{$\overbrace{}^{\text {TEX I }}$} & \multicolumn{2}{|c|}{ PHIL I } & \multicolumn{2}{|c|}{ CR } & \multicolumn{2}{|c|}{ TEX V } & \multicolumn{2}{|c|}{ PHIL II } & \multicolumn{2}{|c|}{ AFR } & \multicolumn{2}{|c|}{ TEX II } & \multicolumn{2}{|c|}{ TEX III } & \multicolumn{2}{|c|}{ TEX IV } \\
\hline & I I & A 2 & A 3 & $\mathrm{~A}_{4}$ & A 5 & A 6 & A 7 & A 8 & B I & B 2 & B 3 & B $_{4}$ & $\mathrm{C}_{\mathrm{I}}$ & $\mathrm{C}_{2}$ & $\mathrm{C}_{3}$ & $\mathrm{C}_{4}$ & $\mathrm{C}_{5}$ & C 6 \\
\hline$F_{1} \mathrm{~S}_{2}$ & 00 & oo & oo & 00 & 00 & OO & $\infty$ & 00 & 00 & $\infty$ & oo & 00 & $\times x$ & $\times \times$ & 00 & $\times \times$ & $x \times$ & $\times \times$ \\
\hline$F_{1}$ & oo & $\infty$ & 00 & $\infty$ & oo & oo & oo & 00 & 00 & oo & oo & oo & $x \times$ & $\times \times$ & 00 & $\times x$ & $\times x$ & $\times \times$ \\
\hline$F_{1} S$ & 00 & 00 & oo & 00 & 00 & oo & $\infty$ & $\infty$ & oo & 00 & oo & oo & $\times x$ & $x \times$ & oo & $x \times$ & $x \times$ & $\times \times$ \\
\hline$F_{1} S$ & oo & oo & oo & ০o & oo & oo & $\infty$ & $\infty$ & $\infty$ & $\infty$ & $\infty$ & $\infty$ & $\times \times$ & $\times \times$ & 00 & $x \times$ & $x \times$ & $\times \times$ \\
\hline$F_{1}$ & oo & oo & oo & 00 & oo & 00 & oo & 00 & Do & $\infty$ & $\infty$ & $\infty$ & $x \times$ & $\times \times$ & $\times \times$ & $\infty$ & $x \times$ & $\times \times$ \\
\hline$F_{1} s$ & oo & ০o & oo & oo & oo & oo & oo & oo & $\infty$ & oo & oo & oo & $\times x$ & $\times \times$ & $<x$ & $\infty$ & $<x$ & $\times \times$ \\
\hline$F_{1}$ & oo & 00 & oo & 00 & oo & oo & oo & 00 & 00 & $\infty$ & oo & $\infty$ & $\times \times$ & $\times \times$ & $<x$ & oo & $<x$ & $x \times$ \\
\hline & $\infty$ & 00 & oo & oo & 00 & 00 & oo & 00 & $\infty$ & $\infty$ & $\infty$ & $\infty$ & $\times x$ & $\times x$ & $x$ & oo & $<x$ & $x \times$ \\
\hline$F_{1}$ & oo & oo & ০o & $\infty$ & $\infty$ & oo & Oo & oo & oo & oo & oo & $\infty$ & $x \times$ & $\times \times$ & $x \times$ & $\infty$ & $x \times$ & $\times \times$ \\
\hline$F_{1}$ & oo & $\infty$ & $\infty$ & oo & $\infty$ & ০০ & 00 & 00 & ০o & $\infty$ & $\infty$ & $\infty$ & $\times x$ & $x \times$ & $x \times$ & $\infty$ & $\times \times$ & $\times x$ \\
\hline
\end{tabular}

$$
x=\text { plasmodial formation. } \quad 0=\text { no plasmodial formation. }
$$

Table 2. Ten clones of the $F_{1}$ generation established from plasmodial genotype $C 2$ $\times C_{4}$ crossed in duplicate to tester clones of the two mating types of each of the nine isolates

\begin{tabular}{|c|c|c|c|c|c|c|c|c|c|c|c|c|c|c|c|c|c|c|}
\hline \multirow{2}{*}{$\begin{array}{c}\text { Clone } \\
\text { numbers }\end{array}$} & \multicolumn{2}{|c|}{ TEX I } & \multicolumn{2}{|c|}{ PHIL I } & \multicolumn{2}{|c|}{ CR } & \multicolumn{2}{|c|}{ TEX V } & \multicolumn{2}{|c|}{ PHIL II } & \multicolumn{2}{|c|}{ AFR } & \multicolumn{2}{|c|}{ TEX II } & \multicolumn{2}{|c|}{ TEX III } & \multicolumn{2}{|c|}{ TEX IV } \\
\hline & I & A 2 & A $3_{3}$ & A 4 & A 5 & A 6 & A 7 & A 8 & B I & B 2 & B 3 & B 4 & $\mathrm{C}_{\mathrm{I}}$ & $\mathrm{C}_{2}$ & $\mathrm{C}_{3}$ & $\mathrm{C}_{4}$ & $\mathrm{C}_{5}$ & $\mathrm{C} 6$ \\
\hline$F_{1} S_{3}$ & Do & 00 & 00 & 00 & 00 & 00 & 00 & ০o & $\infty$ & 00 & 00 & 00 & $\times \times$ & 00 & $\times \times$ & $\times \times$ & $\times \times$ & $\times \times$ \\
\hline$F_{1}$ & $\infty 0$ & $\infty$ & $\infty$ & oo & oo & oo & ০o & oo & oo & oo & oo & oo & $\times \times$ & & $x \times$ & $x$ & $x$ & $x \times$ \\
\hline$F_{1} S$ & oo & $\infty$ & oo & oo & oo & $\infty$ & $\infty$ & $\infty$ & $\infty$ & $\infty$ & $\infty$ & oo & $x \times$ & & $x$ & $x$ & $<x$ & $x \times$ \\
\hline$F_{1} S$ & 00 & $\infty$ & 00 & 00 & 00 & $\infty$ & $\infty$ & ০o & oo & $\infty$ & 00 & $\infty$ & $\times \times$ & & $x \times$ & $x$ & $x$ & $x \times$ \\
\hline$F_{1} S$ & $\infty$ & oo & oo & oo & $\infty$ & $\infty$ & $\infty$ & ০o & oo & $\infty$ & oo & 00 & $x \times$ & & $x \times$ & $x \times$ & $<x$ & $<x$ \\
\hline$F_{1} S$ & $\infty 0$ & $\infty$ & oo & oo & $\infty$ & $\infty$ & $\infty$ & $\infty$ & oo & $\infty$ & $\infty$ & oo & $x \times$ & 00 & $x \times$ & $x \times$ & $x$ & $x \times$ \\
\hline$F_{1}$ & Do & $\infty$ & oo & oo & oo & $\infty$ & $\infty$ & oo & $\infty$ & $\infty$ & $\infty$ & $\infty$ & $\times \times$ & $\times x$ & $\times \times$ & oo & $x$ & $x \times$ \\
\hline & 0 & oo & oo & 00 & $\infty$ & oo & $\infty$ & oo & oo & $\infty$ & 00 & $\infty$ & $\times \times$ & $\times \times$ & $\times \times$ & $\infty$ & $x \times$ & $x \times$ \\
\hline & $\infty 0$ & $\infty$ & $\infty$ & $\infty$ & oo & $\infty$ & $\infty$ & $\infty$ & $\infty$ & $\infty$ & $\infty$ & $\infty$ & $\times \times$ & $\times \times$ & $\times \times$. & oo & $<x$ & $\times \times$ \\
\hline$F_{1} \mathrm{~S}_{7}$ & 00 & $\infty$ & oo & $\infty$ & 00 & $\infty 0$ & $\infty$ & $\infty$ & 00 & $\infty$ & 00 & $\infty 0$ & $\times \times$ & $\times \times$ & $\times \times$ & 00 & $\times \times$ & $\times x$ \\
\hline
\end{tabular}

$$
x=\text { plasmodial formation. } \quad 0=\text { no plasmodial formation. }
$$

Io $F_{1}$ clones were crossed back to each parent clone and also crossed with one tester clone of each mating type of the other eight isolates. Table I shows the results of ten $F_{1}$ clones established from the TEXAS III isolate in such a cross. In this cross the parent clones are represented by alleles $\mathrm{C}_{3}$ and $\mathrm{C}_{4}$. Four of the $F_{1}$ clones crossed with parent clone $\mathrm{C}_{4}$ but not with $\mathrm{C}_{3}$ indicating these four clones were of the same mating type as parent $\mathrm{C}_{3}$. The other six $F_{1}$ clones crossed with the parent clone $\mathrm{C}_{3}$ but not with $\mathrm{C}_{4}$ indicating the remaining six $F_{1}$ clones were of mating type $\mathrm{C}_{4}$. All ten $F_{1}$ clones established from the TEXAS III isolate crossed with mating types $\mathrm{C}$ I, C $2, \mathrm{C} 5$ and $\mathbf{C} 6$ as expected in a multiple allelic system. A similar pattern was obtained for 
the TEXAS IV isolate. The $F_{1}$ clones of the TEXAS $\mathrm{V}$ isolate could be divided into two mating types when crossed back to the two parent clones (A 7, A 8). All $F_{1}$ clones crossed with the tester clones of the other three isolates in group I but failed to cross with clones of groups 2 and 3.

Inter-isolate crosses within group 3 gave a potential of 12 parental plasmodia. These were $\mathrm{C}_{1} \times \mathrm{C}_{3}, \mathrm{C}_{\mathrm{I}} \times \mathrm{C}_{4}, \mathrm{C} \mathrm{I}_{1} \mathrm{C}_{5}, \mathrm{C} \mathrm{I}_{1} \mathrm{C}_{6}, \mathrm{C}_{2} \times \mathrm{C}_{3}, \mathrm{C}_{2} \times \mathrm{C}_{4}, \mathrm{C}_{2} \times \mathrm{C}_{5}$, $\mathrm{C} 2 \times \mathrm{C} 6, \mathrm{C}_{3} \times \mathrm{C}_{5}, \mathrm{C} 3 \times \mathrm{C} 6, \mathrm{C}_{4} \times \mathrm{C}_{5}, \mathrm{C} 4 \times \mathrm{C} 6 . F_{1}$ clones were established from sporangia derived from plasmodia of each of the above types. The clones were then crossed back to the parent clones and to tester clones of both mating types of the other eight isolates. The results of one such cross is given in Table 2 where clones $\mathbf{C} 2$ and $\mathrm{C}_{4}$ were the parental clones. Single-spore isolations were made from sporangia that formed from the resulting plasmodia. The $F_{1}$ clones were divided into two mating types, six crossed with one parent clone $\left(\mathrm{C}_{4}\right)$ but did not cross with the second parent (C 2). The remaining four clones crossed with parent $\mathrm{C}_{2}$ but not with parent $\mathrm{C}_{4}$. All ten $F_{1}$ clones crossed with clones of mating type $\mathrm{C}$, $\mathrm{C}_{3}, \mathrm{C} 5$ and $\mathrm{C} \mathrm{6}$, but did not cross with any clones belonging to the other two incompatibility series. $F_{1}$ clones established from sporangia of the other II parental genotypes gave similar results in that the clones could be segregated into the two mating types comprising the parental genotype. None of the $F_{1}$ clones of the group 3 isolates would cross with clones established from isolates belonging to groups I or 2.

\section{Comparative morphology of the three genetic groups}

A comparative morphological study was made of the nine isolates. Some of the morphological characteristics studied were shape of sporangium, length of stalk, and diameter of spores. Preparation of materials was according to Henney (1967). These included sporangia developed under a variety of environmental conditions to exclude the possibility that the variations were environmentally induced. The diameter and width of the sporangia were measured and plotted as a ratio of diameter of sporangium: width of sporangium. The length of the stalks and diameter of spores were also measured.

The study of stalk length divided the six original isolates into three distinct groups (Henney, I967). Group I had short stalks, group 2 had very long slender stalks, while the stalks of group 3 were intermediate. When this morphological character was studied in the three new isolates they, too, could be placed in two of the three groups corresponding to their respective genetic groups (Fig. 2). The TEXAS $v$ isolate, which crossed with group I, also had short stalks about $0.5 \mathrm{~mm}$. in length, typical of this group. The TEXAS III and TEXAS IV isolates, belonging to genetic group 3, were placed in morphological group 3. The separation from group 2 was not as complete in this case as the stalks were somewhat longer than previously indicated and tended to bridge the gap between groups 2 and 3 .

The ratio of diameter of sporangium: width of sporangium again separated the isolates into morphological groups corresponding to their respective genetic groups (Fig. 3). The isolates comprising group I were all very lenticular and the diameter of the sporangia about 3 times the thickness. The TEXAS $v$ isolate belonged to this group. Group 3 consisted of a much smaller sporangium and was globose to subglobose with the ratio approaching $I \cdot O$ (TEXAS III and TEXAS IV). Group 2 was intermediate, with a 


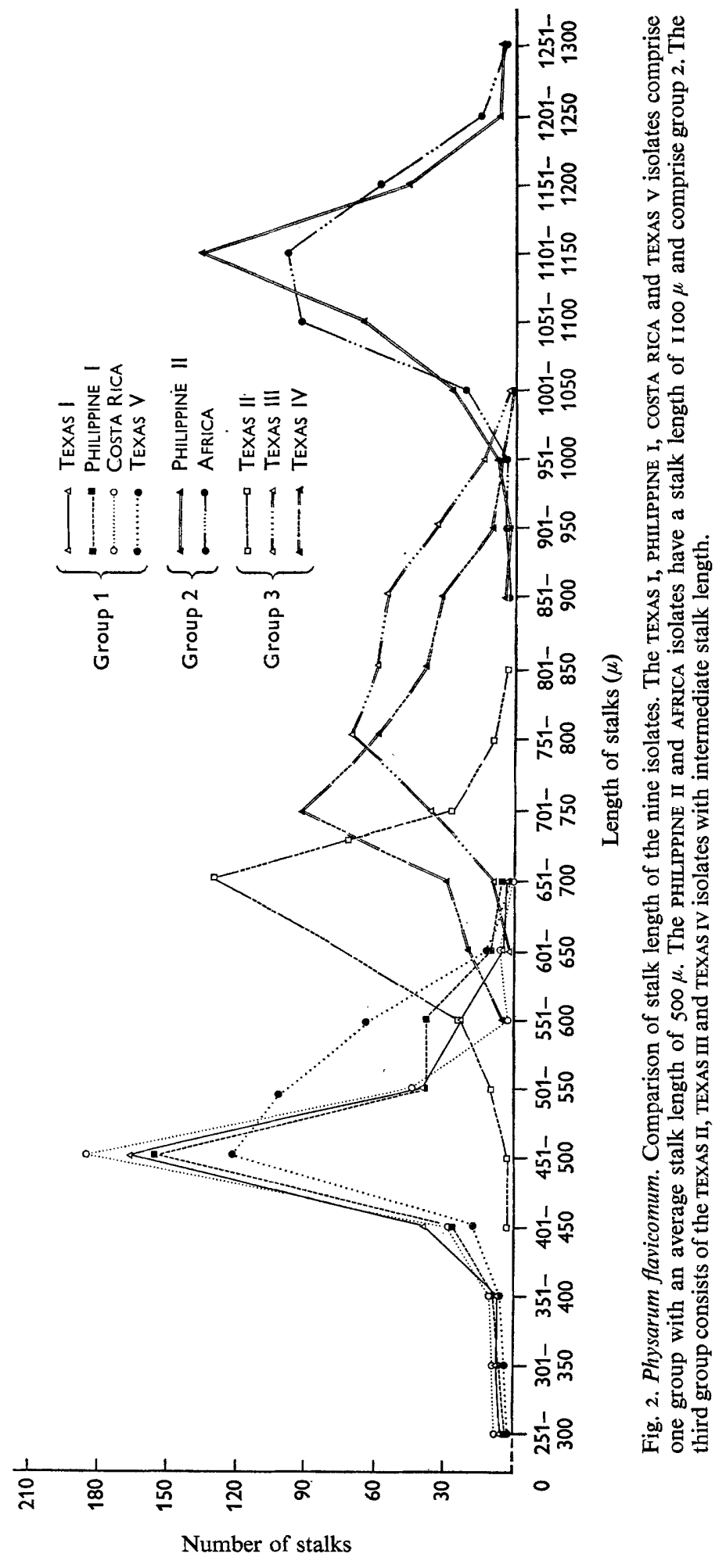



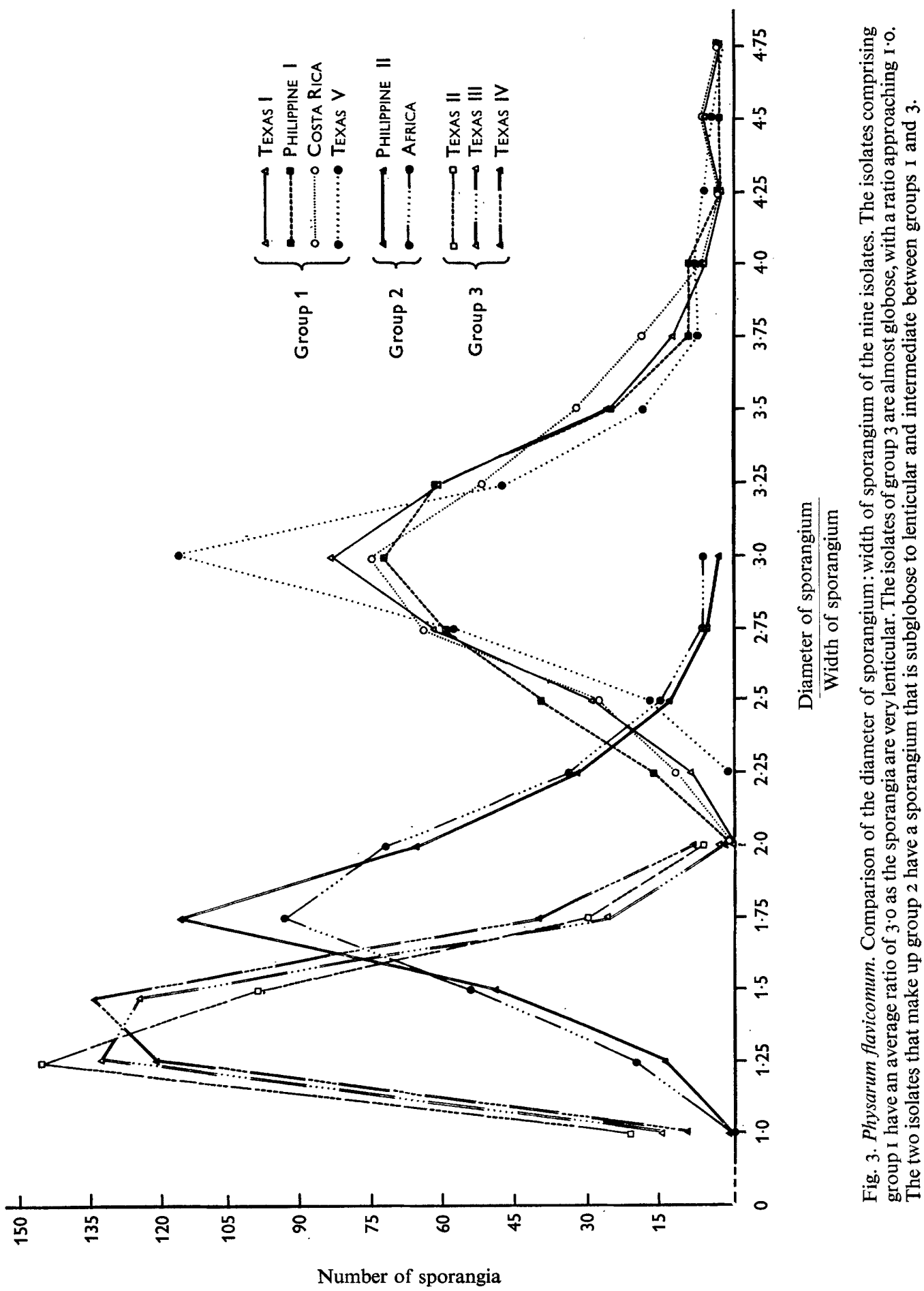


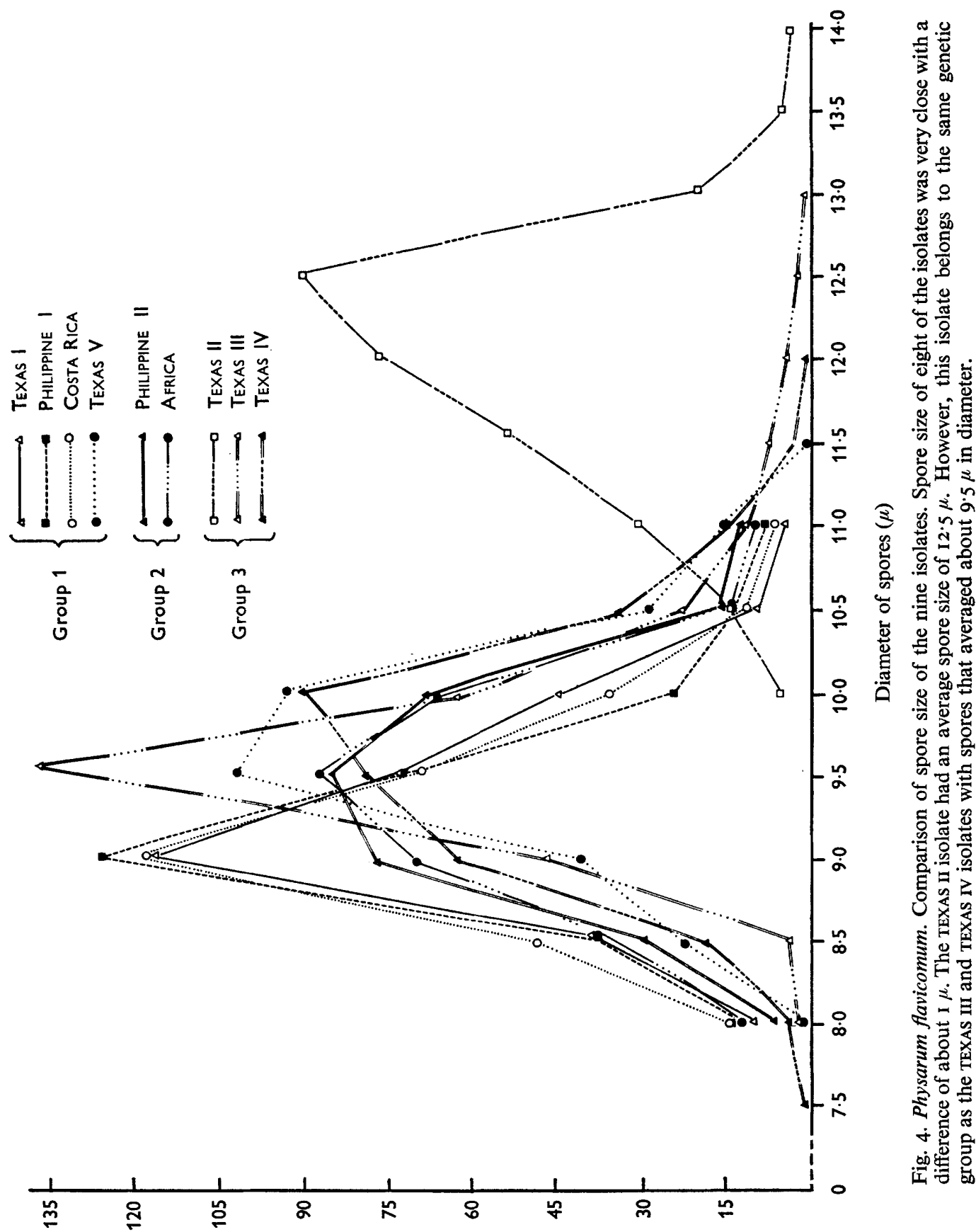

Number of spores 
somewhat larger sporangium than group 3 , and tended to be umbilicate above. Again the two new isolates tended more to combine groups 2 and 3 than to separate them.

The original study indicated the spores of group 3 (this consisted of the single isolate TEXAS II) were much larger than those of groups I and 2. However, the comparative study of TEXAS III and TEXAS IV isolates, which belonged to the same genetic group as the TEXAS II isolate, had spores that were indistinguishable from those of groups I and 2, indicating spore size is variable within group 3, and this character cannot be used to separate group 3 from the other two groups.

The capillitium of the TEXAS $\mathrm{v}$ isolate was characteristic of the group I type (P1. I, fig. I). Here the capillitial threads were sparse and started at the base of the sporangium and extended to the top of the peridium with little or no branching. In contrast the capillitia of groups 2 and 3 were very dense with the threads starting at the base of the sporangium and approximately half-way up, branched profusely and formed a dense network toward the peridium. The capillitia of TEXAS III and TEXAS IV were of this type (Pl. I, fig. 2).

\section{DISCUSSION}

Taxonomic implications immediately became evident when it was found that Physarum flavicomum could be divided into three compatibility groups based on their mating-type reactions. Using the key of Drs G. W. Martin and C. J. Alexopoulos (personal communication: in preparation) all nine isolates used in this study keyed out to P. flavicomum. However, using the older keys of Lister (1925), Macbride \& Martin (I934) and Martin (1949), the isolates comprising group I keyed out to $P$. rigidum except for the absence of the rigid rod-like tubes which characterize that species. Both species belong to a very closely related group called the 'Physarum viride complex' consisting of $P$. viride, $P$. nutans, $P$. rigidum, $P$. bethelii and $P$. flavicomum. All are separated by relatively minute differences. The problem becomes even more complex as it has been impossible to grow $P$. viride, $P$. nutans and $P$. bethelii even in crude culture.

As more studies were made it became more apparent that those isolates comprising group I were different from those comprising groups 2 and 3. This is true genetically, morphologically and physiologically. The shape of the sporangium in group I was large and very lenticular, whereas sporangial shape in groups 2 and 3 tended to be globose to subglobose. The length and type of stalk in group I was short and thick as compared to stalks of the other two groups and was dark below from included refuse matter. The stalk of Physarum flavicomum was free from all inclusions (Lister, I925).

The most important difference separating group I from groups 2 and 3 was that of capillitium. These differences are pictured in Pl. I, fig. I and 2. Macbride \& Martin (1934) stated that the capillitium of Physarum rigidum consisted of sparingly branched threads with long orange-yellow nodes, or consisted almost entirely of slender, rodlike tubes enclosing yellow lime granules. The capillitium of isolates in group I consisted entirely of sparingly branched threads but the slender, rod-like tubes which characterizes this species were never found. It was because of the absence of these calcareous rods that the group I isolates were originally placed in P. flavicomum. However, the description of the capillitium of $P$. flavicomum is given as 'Capillitium a close network of hyaline threads with numerous yellow flat expansions at the axils, often persistent and retaining the form of the sporangium after the dispersion of the 
spores;...'. Whereas this was an accurate description of the capillitia of groups 2 and 3 , it did not describe the capillitium of group $\mathrm{I}$. Since the type of capillitium is considered to be an important taxonomic characteristic in the classification of the myxomycetes (Alexopoulos, I967), the inclusion of isolates with such different capillitial types in the same species seemed to be unwarranted. Capillitia of groups 2 and 3 were indistinguishable.

The morphology of the plasmodium has not been used taxonomically to any great extent in the identification of the myxomycetes. Alexopoulos (1960, 1963, 1966) has recently focused attention on this aspect and has described three general types of plasmodia corresponding to the major groups in the myxomycetes. However, little significance has been placed on the differences in plasmodia within the different groups, mainly because of the variability of the plasmodium under different environmental conditions. Recently, isolates from each of the three before-mentioned groups have been put in pure culture on a partially defined medium (Henney \& Henney, I968), thus enabling growth under controlled conditions. The plasmodial characteristics of the different groups have been constant for about I year and showed distinct differences in morphology and colour.

Another difference separating the three groups was the time required after mating for plasmodial formation to take place. Plasmodial formation in group I occurred 5-6 days after clones of opposite mating types were mixed together. In group 2 the time period for plasmodial formation was nearly 2 weeks, whereas $3-4$ days was sufficient for this process with the isolates comprising group 3 .

It has been decided that the isolates comprising group I are much more appropriately placed in Physarum rigidum than in P. flavicomum because (I) they belonged to different genetic groups, (2) there were differences in the morphology of the sporophores, (3) there were differences in the morphology of the plasmodia in pure culture under controlled environent, and (4) there were physiological differences of time required for plasmodial formation. All these are evidence that these isolates belong to different species. This same reasoning can be applied to those isolates belonging to groups 2 and 3. They, too, belonged to different genetic groups, there were also some differences in the morphology of the sporophore, the plasmodia in pure culture were quite different and the required time for mating was significantly longer for group 2. In this case, however, the morphological differences were much more subtle and the morphological characteristics of the new isolates used in this study tended to bridge the gaps instead of separating them into distinct entities. It is very possible that groups 2 and 3 also belong to different species. After many more isolates are studied a pattern may emerge by which these two groups can readily be identified, but presently we are retaining both groups in $P$. flavicomum as varieties: group $2=P$. flavicomum variety $\mathrm{I}$, group $3=P$. flavicomum variety 2 .

The authors are grateful to Dr Constance Wollman for supplying the isolate of Physarum rigidum and to $\mathrm{Mr} \mathrm{D}$. Jungkind for taking the photographs. This work was supported by University of Houston grant no. F.R.S.P. $67-4$. 


\section{REFERENCES}

Alexopoulos, C. J. (1960). Gross morphology of the plasmodium and its possible significance in the relationships among the Myxomycetes. Mycologia 52, $\mathbf{1}$.

Alexopoulos, C. J. (1963). The Myxomycetes. II. Bot. Rev. 29, I.

Alexopoulos, C. J. (1966). Morphogenesis in the Myxomycetes. In The Fungi. Ed. by G. C. Ainsworth and A. S. Sussman, vol. 2, p. 21 I. New York: Academic Press.

Alexopoulos, C. J. (1967). Taxonomic studies in the Myxomycetes. I. The genus Macbrideola. Mycologia 59, 103.

Collins, O. R. (196r). Heterothallism and homothallism in two Myxomycetes. Am. J. Bot. 48, 674 .

Collins, O. R. (1962). Mating types in the slime mold Physarum pusillum. (Abstr.) Am. J. Bot. 49, 659.

Collins, O. R. (1963). Multiple alleles at the incompatibility locus in the Myxomycete Didymium iridis. Am. J. Bot. 50, 477.

Collrns, O. R. (1965). Homothallic behaviour in two Costa Rican isolates of the slime mold Didymium iridis. (Abstr.) Am. J. Bot. 52, 634.

DEE, J. (1960). A mating-type system in an acellular slime mould. Nature, Lond. 185, 780.

DEE, J. (1966). Multiple alleles and other factors affecting plasmodium formation in the true slime mold Physarum polycephalum Schw. J. Protozool. 13, 6ro.

HenneY, M. R. (1966). The mating type systems in the myxomycete Physarum flavicomum and in the ascomycete Anixiopsis stercoraria. Ph.D. dissertation, University of Texas, Austin.

Henney, M. R. (1967). The mating type system of the Myxomycete Physarum flavicomum. Mycologia $59,637$.

HENNEY, H. R., JUN., \& HENNEY, M. R.(I968). Nutritional requirements for the growth in pure culture of the Myxomycete Physarum rigidum and related species. J. gen. Microbiol. 53, 333 .

KeRR, M. D. \& Sussman, M. (1958). Clonal development of the true slime mold, Didymium nigripes. J. gen. Microbiol. r9, 173.

Lister, A. L. (1925). A Monograph of the Mycetozoa, 3rd ed., revised by G. Lister, London: British Museum (Nat. Hist.).

MACBRIDE, T. H. \& MARTIN, G. W. (1934). The Myxomycetes. New York: Macmillan Co.

Martin, G. W. (1949). The Myxomycetes. N. Am. Flora 1, 1. New York: New York Botanical Garden.

Stosch, H. A. voN (1935). Untersuchungen über die Entwicklungsgeschichte der Myxomyceten. Sexualität und Apogamie bei Didymiaceen. Planta 23, 623.

Wollman, C. (I966). Cultural studies of selected species of Myxomycetes. Ph.D. dissertation, University of Texas, Austin.

\section{EXPLANATION OF PLATE}

Fig. I. Capillitium of Physarum rigidum. The threads start at the base of the sporangium and go to the peridium with little or no branching. The magnification is about $80 \times$.

Fig. 2. Capillitium of Physarum flavicomum. The threads start at the base of the peridium but soon form a profusely branching network. The magnification is about $90 \times$. 
Journal of General Microbiology, Vol. 53, No. 3

Plate I
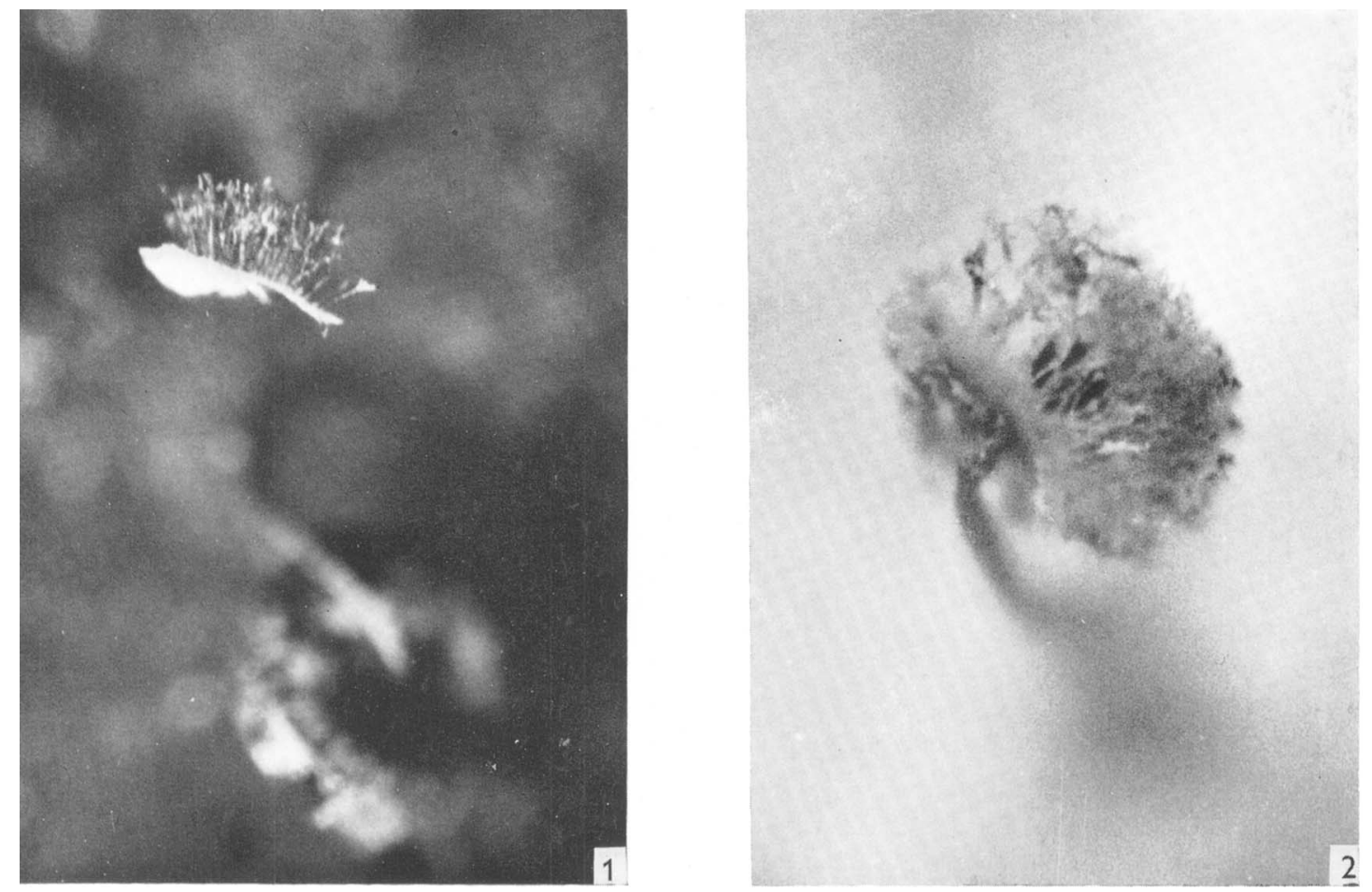